\title{
To compare empirical treatment of uncomplicated UTI in pregnancy
}

\author{
Vipul S. Patel \\ Assistant Professor, Dept. of Obstetrics and Gynecology, Dr. M. K. Shah Medical College \& Research Center, Gujarat, India \\ *Corresponding Author: \\ Email: drvipul2112@gmail.com
}

Received: $17^{\text {th }}$ April, 2018

Accepted: $19^{\text {th }}$ April, 2018

\begin{abstract}
Objectives: To compare empirical treatment with fosfomycin and nitrofurantoin in uncomplicated lower urinary tract infection during pregnancy.

Materials and Methods: Patients were recruited from OPD of obstetrics and gynecology presenting with symptoms of lower (UTIs) urinary tract infection. Patients were divided randomly alternately in two groups. Fosfomycin therapy was given in group $1(n=60)$ and seven days nitrofurantoin therapy was given in group $2(n=60)$. Patients were evaluated for symptoms improvement, compliance, side effects and cost of treatment.

Results: Efficacy of fosfomycin on day 7 post treatment is $98 \%$ compare to nitrofrantoin I s83.3\%. Side effect is $15 \%$ in fosfomycin group as compare to $28.3 \%$ in nitrofurantoin group. Compliance is $100 \%$ in fosfomycin group as compare to $96.6 \%$ in nitrofrantoin group. Cost of therapy in fosfomycin group is 300 Rs average while 170 Rs in nitrofurantoin group.

Conclusion: Fosfomycin is first line empirical treatment of uncomplicated UTIs during pregnancy because it is safe, effective, less resistance, higher patient's compliance with fewer side effect and equal cost of therapy.
\end{abstract}

Keywords: Pregnancy, Cystitis, Nitrofurantoin, Fosfomycin, UTIs.

\section{Introduction}

Urinary tract infections (UTIs) are commonest bacterial infection in pregnancy. ${ }^{1}$ It occurs in 3 to $10 \%$ of pregnancy and requires antibiotic usage. Uncomplicated UTIs is defined as acute cystitis occurring with no abnormalities of urological tract and other medical conditions, and it is characterized by symptoms of burning of micturation, dysuria, urgency, frequency, suprapubic pain and haematuria. ${ }^{2} \mathrm{E}$ coli is the commonest bacteria responsible for uncomplicated UTIs $(82.5 \%)$. Other bacteria like proteus mirabilis, klebsiella pneumonia, staphylococcus saprophyticus and entococcus fecalis are also found in uncomplicated UTIs. $^{3}$

There is increased resistance to various antimicrobials. More than one fourth of e.coli strains causing acute cystitis are now resistant to common antimicrobials such as amoxicillin, sulfa drugs, co trimoxazole and cephalexin. Resistance to fluroquinolones is also rising. In an Indian study by Akram et al., it was reported that ciprofloxacin resistance rate were ranging from 47 to $69 \%$ among the gram negative organisms. ${ }^{4}$

Following factors are considered for selecting antibiotic for the treatment of acute cystitis are efficacy, resistance rates, side effects, selection of drug resistant organisms and development of colonization with multidrug resistant organisms, cost and availability of drug.

Fosfomycin, a natural antibiotic compound produced by several Streptomyces and Pseudomonas species. Fosfomycin is a bactericidal antibiotic that inhibit the initial step in cell wall synthesis. Adherence of bacteria to uroepithelial cells is reduces. Fosfomycin has ability to inhibit the formation and even promote disruption of E. coli biofilm present in the bladder in acute cystitis. This help in prevention of recurrence and development of chronic infection. Cross resistance between fosfomycin and other antibacterial agents is almost nil.

Fosfomycin shows a broad spectrum powerful bactericidal activity against gram negative such as Escherichia coli, klebsiella pneumonia, proteus mirabilis and entrobacter cloacae. It is also very effective against gram positive bacteria like staphylococcus aureus, streptococcus pneumonia, entrococcus faecalis and entrococcus faecium. ${ }^{5}$

\section{Materials and Methods}

Total one hundred and twenty patients from OPD department of OB \& GY of Dr. M.K. Shah Medical College and Research Institute. Pregnant women from 12 to 36 weeks of pregnancy with symptoms of burning of micturation, dysuria, urgency, frequency, suprapubic pain (uncomplicated lower UTIs.) were included in study. Approval of ethical committee was taken.

\section{Exclusion Criteria}

1. Congenital urogenital anomalies

2. Hydronephrosis

3. Renal stones

4. Diabetes.

5. Patients with Imunocompromised status.

6. Patients of pylonephritis with loin pain with high grade fever.

7. Patients in whom oral medication is not possible due to severe nausea and vomiting. 
Patients were divided randomly alternatively in two groups.

Group 1 received single dose of $3 \mathrm{gm}$ fosfomycin trometamin sachet dissolved in half glass of water at bed time after empting of bladder.

Group 2 received oral nitrofurantoin sustain released capsules two times a day for seven days

Both groups were evaluated after seven days of treatment for symptoms relieved, side effects and compliances. All patients were undergone urine routine micro after seven days.

\section{Results}

One hundred and twenty patients suffering from UTIs (cystitis) were enrolled in this study. All patients were received treatment with either fosfomycin or nitrofurantoin.

Details of patients enrolled. All patients were matched in term of age, gravidity, weeks of pregnancy, and B.M.I.

\section{Table 1}

\begin{tabular}{|l|c|c|}
\hline & Group 1 & Group 2 \\
\hline Age & $20.2-32.6$ & $20-33.2$ \\
& Mean Age 25.4 \pm 2.8 & Mean Age 25.7 \pm 2.7 \\
\hline Gravidity & $1-4$ & $1-4$ \\
& Mean 1.7 \pm 0.4 & Mean 1.6 \pm 0.3 \\
\hline Weeks of Pregnancy & $20.2-35.4$ & $19.8-36$ \\
& Mean Week 28 \pm 4.2 & Mean Week 29 \pm 3.2 \\
\hline B.M.I & $19.5-35$ & $19.5-35$ \\
& Mean BMI 24.2 \pm 3.4 & Mean BMI 24.5 \pm 2.8 \\
\hline
\end{tabular}

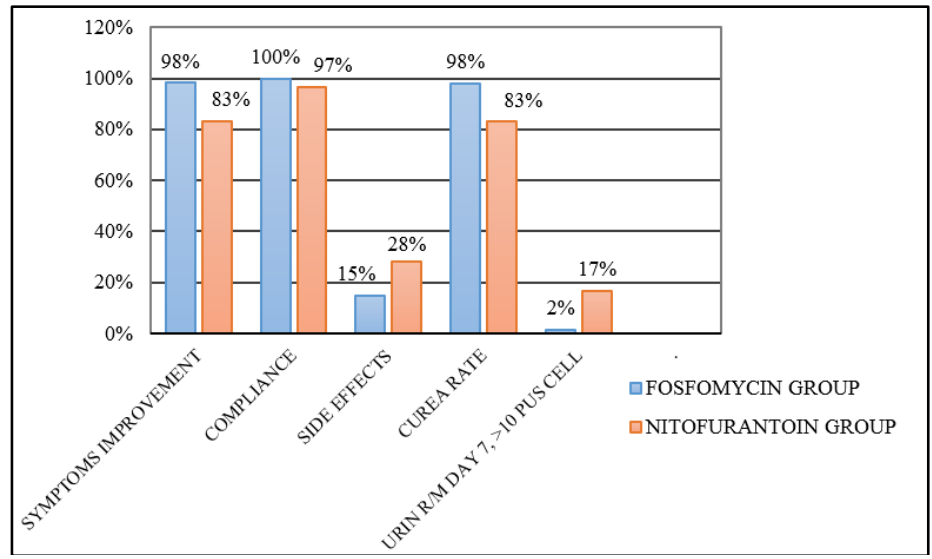

\section{Fig. 1: Parameter to be compare}

Efficacy by symptoms improvement at day 7 post treatment of two drugs was evaluated. In fosfomycin group $59(98.3 \%)$ patients were symptoms free compare to nitrofurantoin group $50(83.3 \%)$ patients.

In urine analysis after seven days treatment in fosfomycin group, 15-20 pus cells were found in one (2\%) patients and 0-10 pus cells were found in 59 (98\%) patients. Nitrofurantoin group 15-20 pus cells were found in $10(17 \%)$ patients and $0-10$ pus cells were in $50(83 \%)$ patients.

Side effects were observed in 9 patients (15\%) in fosfomycin therapy and 17 patients (28.3\%) in 7 days nitrofurantoin treatment. Common side effects are diarrhea, nausea, vomiting and vaginitis in fosfomycin therapy. While in nitrofurantoin group is diarrhea, dyspepsia, and nausea and vomiting.

The cure rate in fosfomycin group is $98 \%$ while in nitrofurantoin group is $83 \%$.
Compliance was $100 \%$ in fosfomycin group as it is single dose and $58(96.6 \%)$ in nitrofurantoin group due to multidose and side effects of drug.

Cost of single dose fosfomycin therapy is approximately $300 \mathrm{Rs}$ as compare to cost of nitrofurantoin treatment for seven days is 170 Rs. Though the cost of fosfomycin is higher than nitrofurantoin but single dosing with high compliance and comparable result with fewer side effects it becomes drug of choice. 


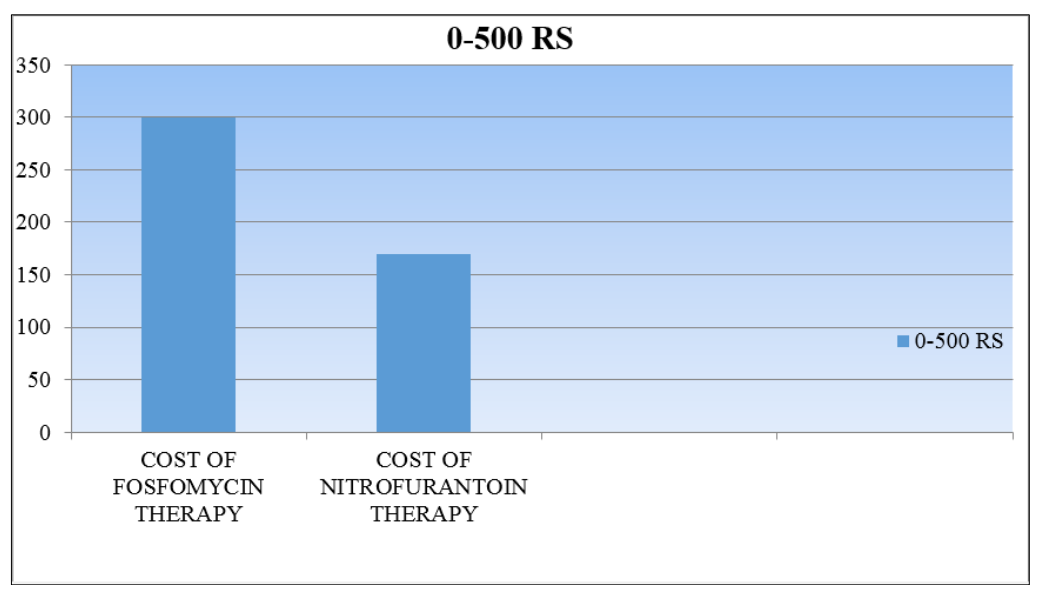

Fig. 2

\section{Discussion}

UTIs are commonest bacterial infection in women. It occurs in $25-30 \%$ of women between 20-40 years' of age and incidence increases directly with sexual activity and child-bearing. $60 \%$ women will develop an episode of UTIs in their lives and one third of those will have a recurrence within a year. ${ }^{6}$

UTIs during pregnancy must be effectively treated to prevent complications associated with it like pyelonephritis, preterm rupture of membrane, preterm labour, chorio-amnionitis, and neonatal septicemia. Repeated urinary tract infection and its treatment with long courses of antibiotic treatment resulted in highly antibiotic resistant bacteria and multi-drug resistant organisms. Urine culture and sensitivity test for diagnosis of UTIs is time consuming and coasty. So empiric treatment for UTI should be consider which has broad spectrum and low resistance with better compliance and cost-effectiveness. So nitrofurantoin and fosfomycin are first line drug of choice for empiric therapy.

Following are evidence supporting use of single dose fosfomycin in UTIs.

The Italian study by Zinner $S$. et al. who randomized 153 UTIs patients with pregnancy to receive fosfomycin (FT) as a single dose of $3 \mathrm{~g}$ and 138 such patients to receive therapy with pipemidic acid (PA), $400 \mathrm{mg}$ B.I.D. for seven days. Infection was eradicated in $147(96 \%)$ of FT and $129(94 \%)$ of PA patients. Similar recurrence rates occurred. Minimal side effects (mostly nausea and dyspepsia) occurred (9\%) FT; $15 \%$ PA). Single-dose FT is equivalent to conventional treatment with PA. ${ }^{7}$

Therapeutic success was achieved by Krcmery S. in $95.2 \%$ of the patients treated with fosfomycintrometamol compare $90.0 \%$ of those treated with ceftibuten. The treatment of acute cystitis in pregnant women using a single-dose of fosfomycin trometamol was equally effective as the 3-day course of oral ceftibuten. Both regimens were well tolerated with only minor adverse effects. ${ }^{8}$
In studies by Reeves DS (two multicentric) fosfomycin trometamol in a $3 \mathrm{~g}$ (as fosfomycin) single dose was given in 250 patients; 197 patients were given one of three other agents. Cure rates for fosfomycin trometamol were $77-94 \%$ (68-94\% for other agents), which was satisfactory in an infection which is sometimes difficult to eradicate. ${ }^{9}$

Usta, T.A., Dogan, O., Ates, U., et al. compare the efficacy of fosfomycin trometamol, amoxicillin clavulanate, cefuroxime axetil antibiotics, and assess the difference in compliance, in the treatment of urinary tract infections during pregnancy. The treatment groups were similar significantly in terms of demographics, clinical success rate, microbiological cure rate, or adverse effects. Higher drug compliance was seen in the fosfomycin trometamol group $(P<0.05) .{ }^{10}$

Keating, G.M. et al show single-dose fosfomycin trometamol had similar efficacy to a 5-day course of cefuroxime axetil or a 7-day course of amoxicillin/clavulanic acid in pregnant women with asymptomatic bacteriuria, and similar clinical and/or bacteriological efficacy to a 5-day course of cefuroxime axetil or amoxicillin/clavulanic acid or a 3-day course of ceftibuten in pregnant women with a lower UTI. Single-dose fosfomycin trometamol was generally well tolerated, with gastrointestinal adverse events (e.g. diarrhoea, nausea) reported most commonly. In conclusion, single-dose fosfomycin trometamol is an important option for the first-line empirical treatment of uncomplicated lower UTIs. ${ }^{11}$

\section{Conclusion}

Because of higher efficacy, safety, low resistance, less side effects, better compliance and comparable cost of treatment with single dose fosfomycin in uncomplicated UTIs during pregnancy had replace other antibiotic regimen during pregnancy. Fosfomycin now become first line of treatment for UTIs during pregnancy.

\section{Conflict of Interests: None.}




\section{References}

1. Colgan, R. and Williams, M. Diagnosis and treatment of acute uncomplicated cystitis. American family physician, 84, 771-776.

2. Hotchandani R et al., Urinary Tract infections in women. Indian Journal of Clinical Practice. 2012:23(4):187-192.

3. Gupta. K., Hooton, T.M., Naber, K.G., et al. (2011) international clinical practice guidelines for the treatment of acute uncomplicated cystitis.

4. Akram $\mathrm{M}$ et al., Etiology and antibioticresistance patterns of community acquired urinary tract infection JNMC hospital Aligarh. Ann Clin Microbiol Antimicrob. 2007;6:4.

5. Novefos. Product monograph sun pharma spectra division.

6. Mittal S et al., Fosfomycin use in Multi Drug Resistant Uropathogenic E. coli. Infectious disorders-Drug Targets. 2015;15(3)196-201.

7. Zinner S (1990) Fosfomycin trometamol versus pipemidic acid in the treatment of bacteriuria in pregnancy. Chemotherapy. 36(Suppl 1):50-52.

8. Krcmery S, Hromec J, Demesova D (2001) Treatment of lower urinary tract infection in pregnancy. Int $J$ Antimicrob Agents. 17:279-282.

9. Reeves DS (1992), Treatment of bacteriuria in pregnancy with single dose fosfomycin trometamol: a review. Infection. 20(Suppl 4):S313-S316.

10. Usta, T.A., Dogan, O., Ates, U., et al. (2011) Comparison of Single-Dose and Multiple-Dose Antibiotics for Lower Urinary Tract Infection in Pregnancy. International Journal of Gynecology \& Obstetrics. 114, 229-233.

11. Keating, G.M. (2013) Fosfomycin Trometamol: A Review of Its Use as a Single-Dose Oral Treatment for Patients with Acute Lower Urinary Tract Infections and Pregnant Women with Asymptomatic Bacteriuria. Drugs. 73, 1951-1966.
12. Lumbiganon, P., Villar, J. and Laopaiboon, M. (2009) One-Day Compared with 7-Day Nitrofurantoin for Asymptomatic Bacteriuria in Pregnancy: A Randomized Controlled Trial. Obstetrics \& Gynecology. 113, 339-345

13. Guinto, V.T., De Guia, B., Festin, M.R. and Dowswell, T. (2010) Different Antibiotic Regimens for Treating Asymptomatic Bacteriuria in Pregnancy. The Cochrane Database of Systematic Reviews, No. 9, CD007855.

14. Unlu, B.S., Yildiz, Y., Keles, I., Kaba, M., Kara, H., Tasin, C., Erkilinc, S. and Yildirim, G. (2014) Urinary Tract Infection in Pregnant Population, Which Empirical Antimicrobial Agent Should Be Specified in Each of the Three Trimesters? Ginekologia Polska, 85, 371-376.

15. Sabharwal ER et al. Fosfomycin: an alternative therapy for the treatment of UTI amidst escalating antimicrobial resistance. Journal of Clinical and Diagnostic Research. 2015;9(12):6-9.

16. Minassian MA et al. A comparison between single dose fosfomycin and a 5 day course of trimethoprim in the treatment of uncomplicated lower urinary tract infection in women. International Journal of Antimicrobial Agents. 1998;10;39-47.

17. Stein GE et al, Comparision of single dose fosfomycin and a 7 day course of nitrofurantoin in female patients with uncomplicated urinary tract infection. Clinther. 1999;21(11):1864-72.

How to cite this article: Vipul S. Patel. To compare empirical treatment of uncomplicated UTI in pregnancy. Ind J Obstet Gynecol Res. 2018;5(3):361-364. 